Journal of Clinical Investigation

Vol. 41, No. 7, 1962

\title{
THE METABOLISM OF RING-LABELED OROTIC ACID IN MAN
}

\author{
By S. M. WEISSMAN, A. Z. EISEN, H. FALLON, M. LEWIS ANd M. KARON
}

(From the Metabolism Service, Dermatology and Medicine Branches, National Cancer Institute, Bethesda, Md.)

(Submitted for publication January 19, 1962; accepted March 29, 1962)

Considerable knowledge concerning purine metabolism has been obtained from chemical and isotopic studies of urinary uric acid. Corresponding information in man is lacking for the closely related field of pyrimidine metabolism. The availability of ring-labeled orotic acid as a specific pyrimidine precursor (1), together with the presence of pseudouridine (5-ribosyluracil) and uracil, as specific end products of pyrimidine metabolism in man, in conveniently assayable amounts in human urine $(2-4)$, makes it possible to conduct certain kinetic studies. In the present investigation the metabolism of ring-labeled orotic acid and the time course of isotope incorporation into urinary pseudouridine and uracil were studied in order to develop methods of measurement and to obtain values for the whole-body rate of pyrimidine synthesis as well as for the turnover rate of certain ribonucleic acid fractions.

\section{METHODS AND EXPERIMENTAL PLAN}

Uracil and pseudouridine were isolated from 15-minute samples of urine by a previously described procedure employing column chromatography in a mixed boratechloride gradient (4). Specimens were concentrated and desalted by absorption on alkaline DOW-1 formate and elution with $0.1 \mathrm{~N}$ formic acid, when necessary for paper chromatography or counting of low specific activity samples (5). Descending paper chromatography was performed by standard techniques on Whatman no. 1 paper with isopropanol:acetic acid:water $(60: 30: 10)$ as the solvent (4). Serial $2 \mathrm{~cm}$-strips were cut from the paper and eluted with water, and the radioactivity in the eluates determined by counting at infinite thinness on stainless steel cups in a low-background (less than $2 \mathrm{cpm}$ ) gasflow counter. Pseudouridine and uracil specimens were counted in a liquid scintillation counter, after dissolving in a dioxane-containing solvent (6). Appropriate standards were counted in the same systems.

A fraction of urine containing orotic acid was separated by DOW-1 formate column chromatography, by procedures described elsewhere (7). This fraction was purified on paper chromatograms with methyl acetate:water: formic acid $(65: 35: 5)$. The orotic acid was then eluted with water and its specific activity determined.
Total plasma and urine radioactivity was determined by first digesting the plasma or urine with an equal vol of $5 \mathrm{~N} \mathrm{KOH}$ for 2 hours at $70^{\circ} \mathrm{C}$, decolorizing with 1 to 2 drops of hydrogen peroxide, and counting $0.2 \mathrm{ml}$ of this digest in $20 \mathrm{ml}$ of the toluene-methanol solution (8). In all cases counting efficiency was determined by the addition of internal standards.

Alternatively, radioactivity of urine was determined by plating serial dilutions of each urine specimen on stainless steel cups, and extrapolating the count rates to infinite thinness. After the first 8 days, the count rate in total urine was generally too low to be conveniently determined directly. Extrapolations of total urine radioactivity were based on the assumption that total urine radioactivity had a constant ratio to urinary pseudouridine activity, since such parallelism was observed in days 3 through 8 , and urinary pseudouridine constituted about one-third of total urine radioactivity during this period.

Timed expiratory air samples were collected in rubber balloons. The carbon dioxide was trapped by bubbling the air through $0.5 \mathrm{~N}$ sodium hydroxide. Barium hydroxide was added, barium carbonate collected by filtration, dried overnight at $110^{\circ} \mathrm{C}$, weighed, pulverized, and a weighed aliquot counted in a liquid scintillation counter after suspension in a thixotropic gel (9). Relative effciency of this counting method had been previously determined in this laboratory (9).

Patients employed in this study were selected because of the presence of an inoperable but slowly progressing malignancy and normal hepatic and renal function. None of the subjects lost any weight during the first 6 weeks of the study. All subjects were maintained on a purinefree, 70-g protein, isocaloric diet for 5 days before and at least 12 days after the orotic acid infusion. The selection of this diet was made to simplify the urinary chromatogram and to limit the exogenous supply of pseudouridine and other pyrimidines.

Urine and expired-air samples were collected at progressively increasing intervals during the first 2 days, daily thereafter for about 1.5 to 2 weeks, and once or twice weekly thereafter when possible, as indicated in the graphs (Figures 1-4).

Patients I, II, and III received an intravenous infusion of $50 \mu \mathrm{c}$ of $6-\mathrm{C}^{14}-, 2-\mathrm{C}^{14}$, and $2-\mathrm{C}^{14}$-orotic acid (2 to $4 \mu \mathrm{c}$ per $\mu$ mole), respectively, over a period of 15 minutes. Patient IV received $35 \mu \mathrm{c}$ of $6-\mathrm{C}^{14}$-orotic acid intravenously over 90 minutes. Patient $I$ had frequent plasma samples drawn over the first 3 hours for determination of radioactivity, in order to obtain an estimate of minimal renal clearance rates for orotic acid. Simultaneous en- 

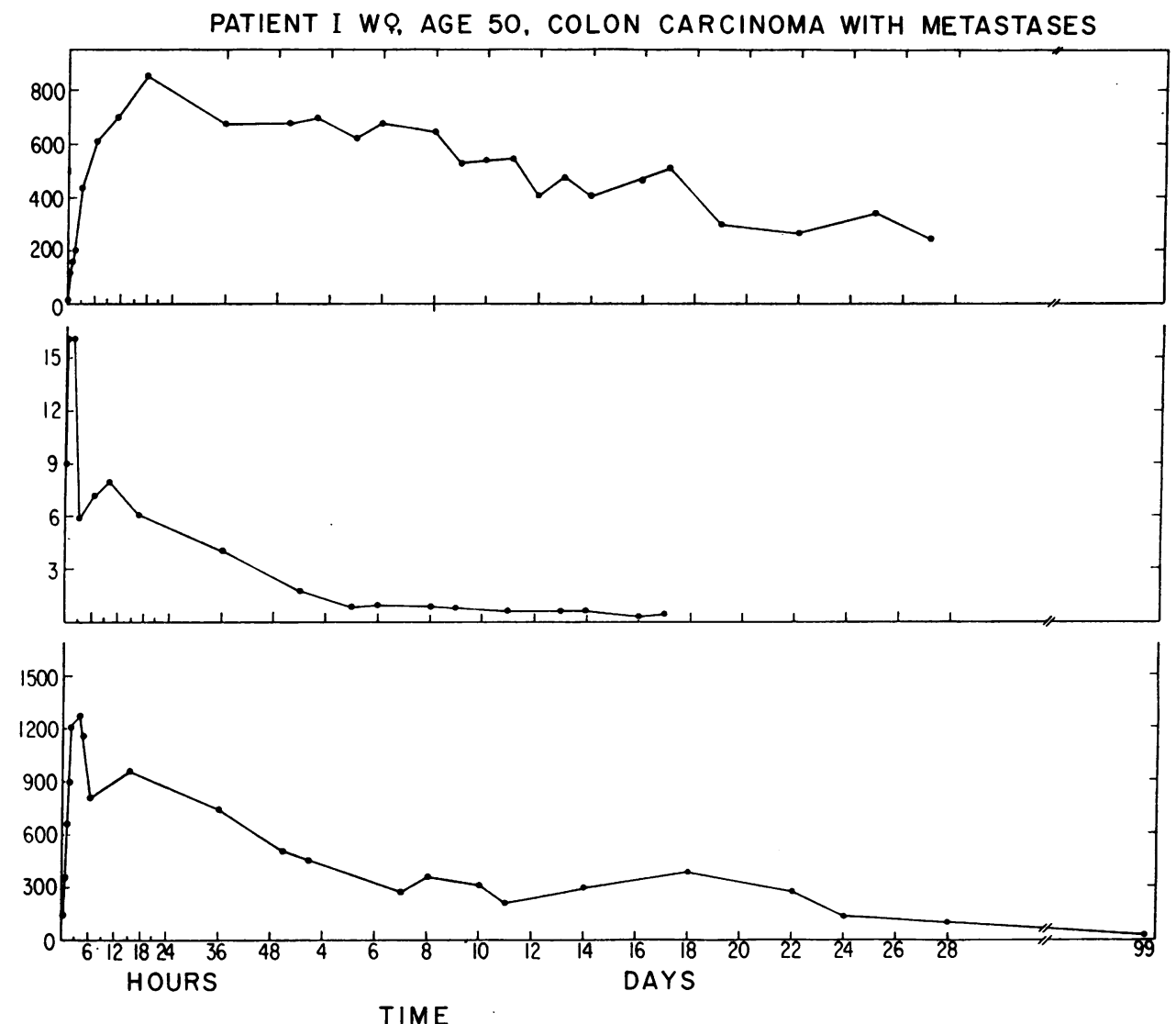

FIG. 1.

dogenous creatinine clearance was measured by standard methods.

Rat liver was examined for acid-soluble pseudouridine compounds. Twelve $\mathbf{g}$ of liver was homogenized and treated with $0.5 \mathrm{~N}$ cold perchloric acid, centrifuged, and the supernate neutralized with $2 \mathrm{~N} \mathrm{KOH}$. It was then treated with sufficient rattlesnake venom and bacterial alkaline phosphatase in $0.05 \mathrm{M}$ Tris buffer, $\mathrm{pH} 8.0$, to convert nucleotide derivatives to nucleosides. The extract was processed by column chromatography in the same manner as the urine samples. The eluates from the uridine and pseudouridine areas of the chromatogram were desalted and carefully examined for pseudouridine by paper chromatography. This was done also with the liver of a rat previously given a total of $200 \mu \mathrm{c}$ of $2-\mathrm{C}^{14}-$ orotic acid in six intraperitoneal doses over 24 hours. Both radioactive and ultraviolet-absorbing materials were sought for and neither was found. The sensitivity of this approach is such that acid-soluble pseudouridine derivatives corresponding to 1 part in 500 of the acid-soluble uridine derivatives would have been readily detected.

A pseudouridine standard was prepared from commercial yeast ribonucleic acid by the methods of Cohn (10). All other chemicals, rattlesnake venom, and Escherichia coli alkaline phosphatase were commercial products.
RESULTS

All radioactivity emerging from the borate columns in the uracil area migrated as uracil when chromatographed on paper. After the first 2 hours, all radioactivity in the pseudouridine area of the column chromatogram behaved like pseudouridine on paper. The earliest urine specimens contained a radioactive compound, generally not associated with detectable amounts of ultravioletabsorbing material, that emerged just ahead of pseudouridine during column chromatography. In order to get accurate pseudouridine specific activities on these specimens, it was necessary, prior to assay, to purify pseudouridine further by paper chromatography.

As shown in Figure 5, when the orotic acid was administered over 15 minutes, the first few urine specimens had an excess of radioactivity, presumably owing to spillage of orotic acid. In Patient I all detectable radioactivity had disappeared from the orotic acid-containing fraction of the appro- 
PATIENT II W\%, 33. ADENOCARCINOMA STOMACH WITH METASTASES
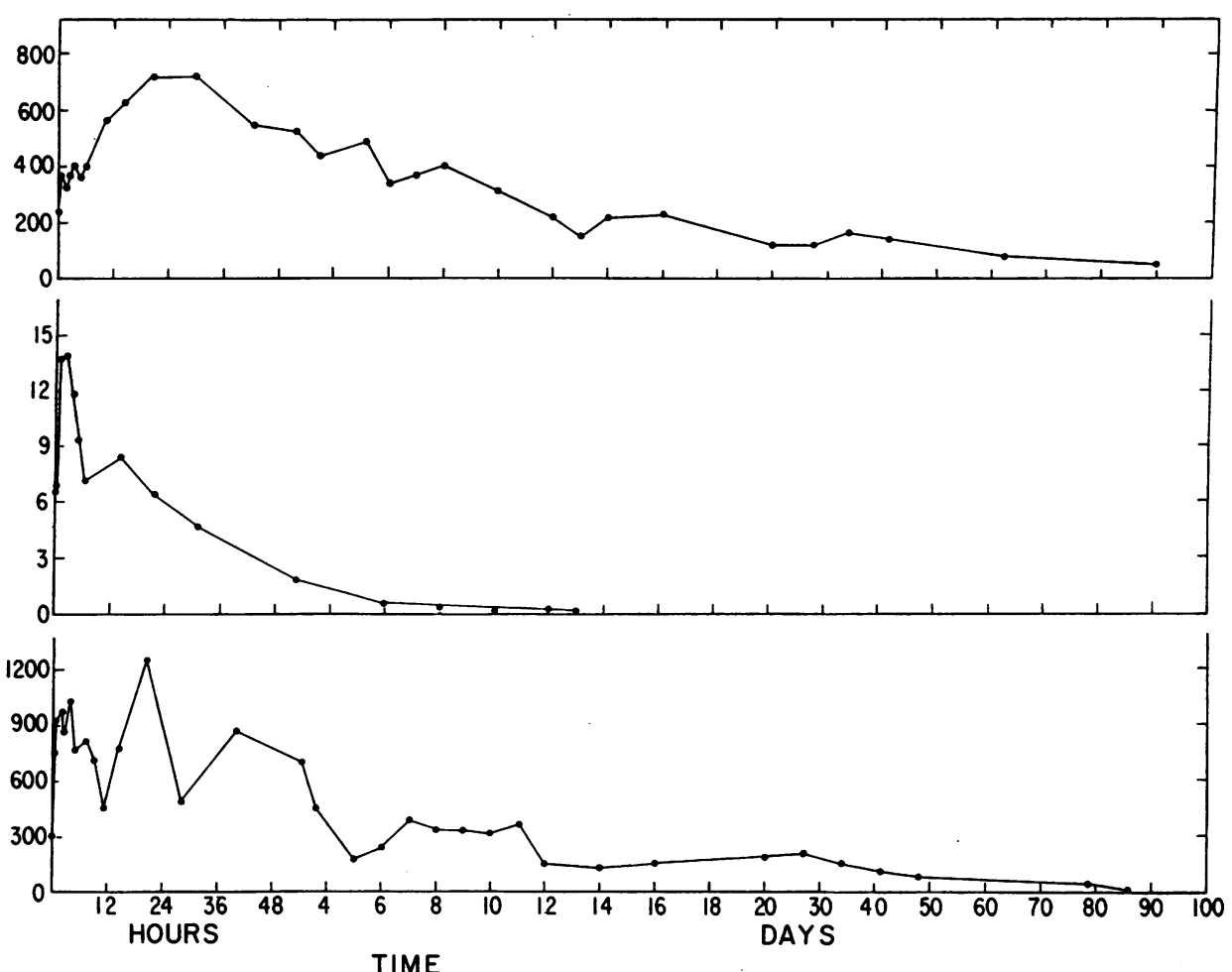

FIG. 2.

PATIENT III W. O. 53, ADENOCARCINOMA COLON WITH METASTASES
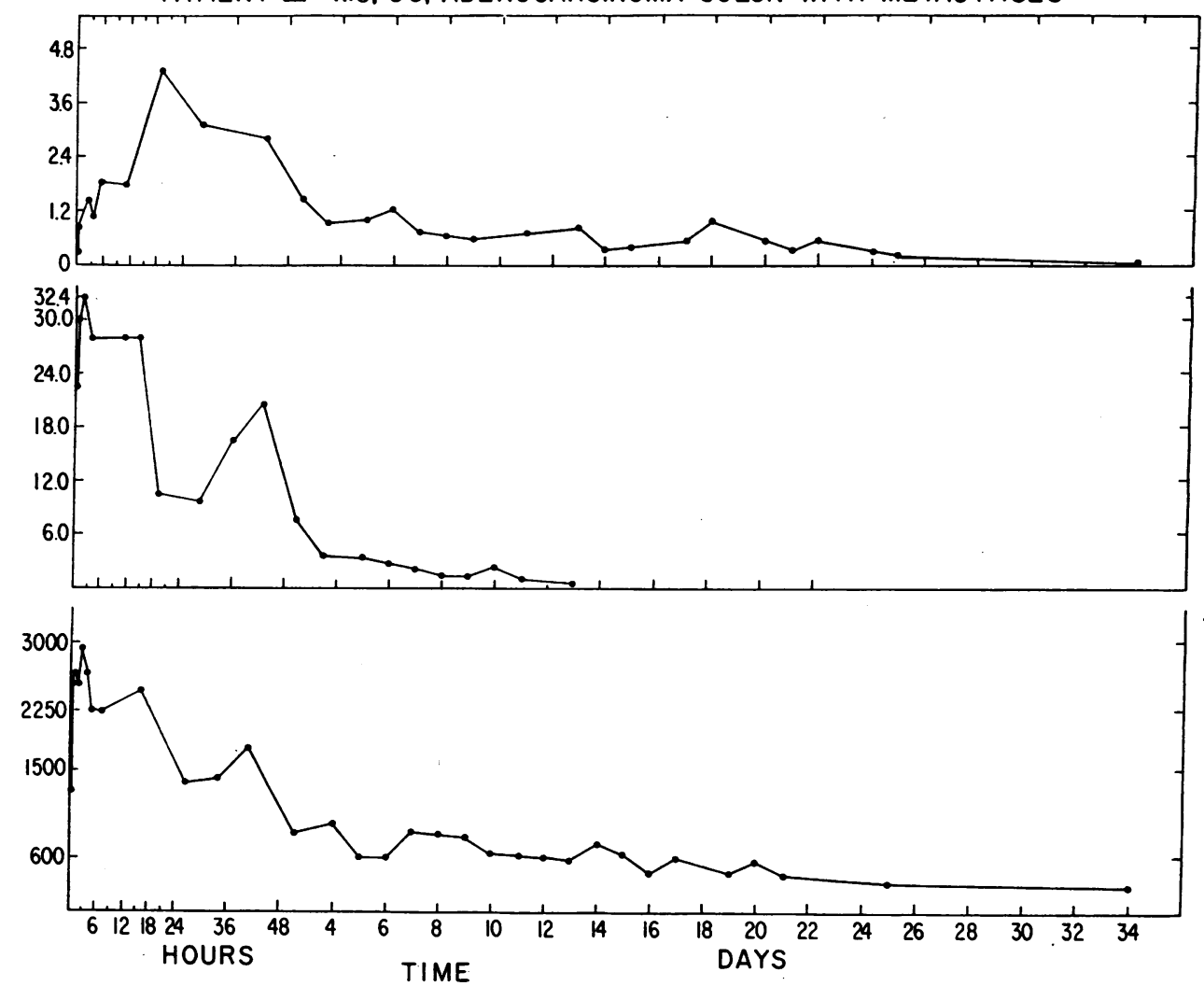

Fig. 3. 
priate column chromatogram by the end of 3 hours after administration of the isotope. Patient IV, who was given the orotic acid infusion more slowly (over a 90 -minute period), did not show an early excess of radioactivity in her urine. The similarity of the general form of the specific activity curves in this and the other studies suggests that the chemical amount of orotic acid given to

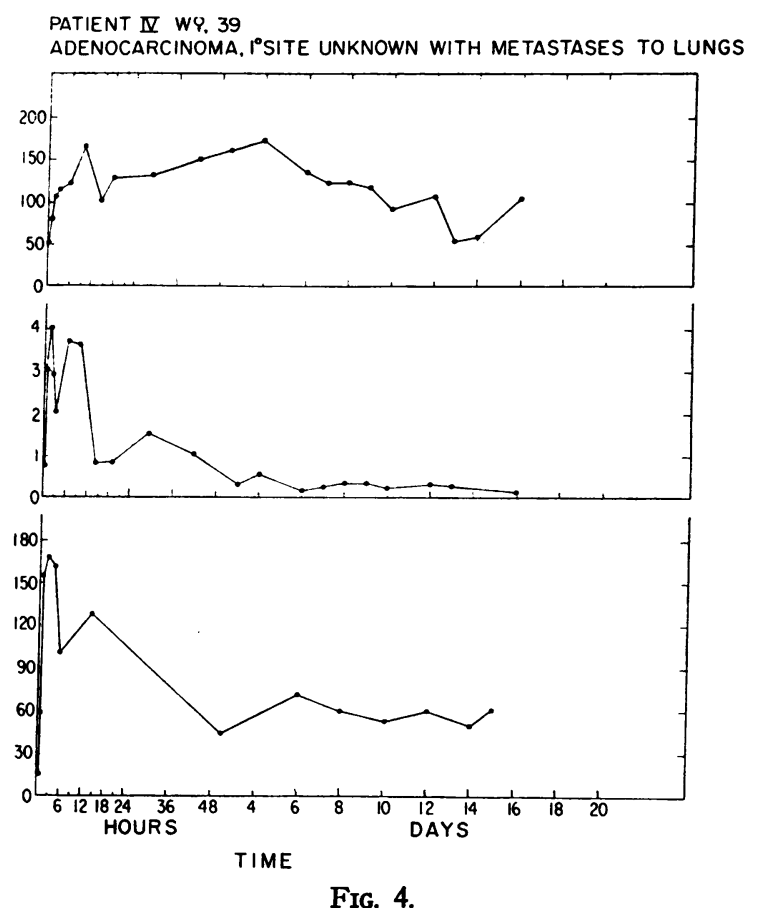

Figs. 1-4. Time course OF SPeCific ACtivity OF URINARY PSEUDOURIDINE AND URACIL AND OF RESPIRATORY $\mathrm{CO}_{2}$ AFTER THE ADMINISTRATION OF ISOTOPICALLY-LABELED OROTIC ACID. Upper graph ordinate: counts per minute per $\mu$ mole urinary pseudouridine $\left(\times 10^{-8}\right.$ but for Patient III, $\left.\times 10^{-6}\right)$. Middle graph ordinate: counts per minute per $\mu$ mole urinary uracil $\left(\times 10^{-6}\right)$. Lower graph ordinate: counts per minute per gram barium carbonate derived from respiratory $\mathrm{CO}_{2}$. Patient $\mathrm{I}$ received $50 \mu \mathrm{c} 6-\mathrm{C}^{14}-$ orotic acid over 15 minutes; Patients II and III received $50 \mu \mathrm{c} 2-\mathrm{C}^{14}$-orotic acid over 15 minutes; Patient IV received $35 \mu \mathrm{c} 6-\mathrm{C}^{14}$-orotic acid over 90 minutes.

Patients I-III did not significantly exceed tracer levels.

The data in Table I indicate that total renal clearance of nonprotein-bound radioactive material approaches the simultaneous clearance of creatinine. In these very early specimens virtually all the urine radioactivity was due to orotic acid, so that these figures represent minimal clearance

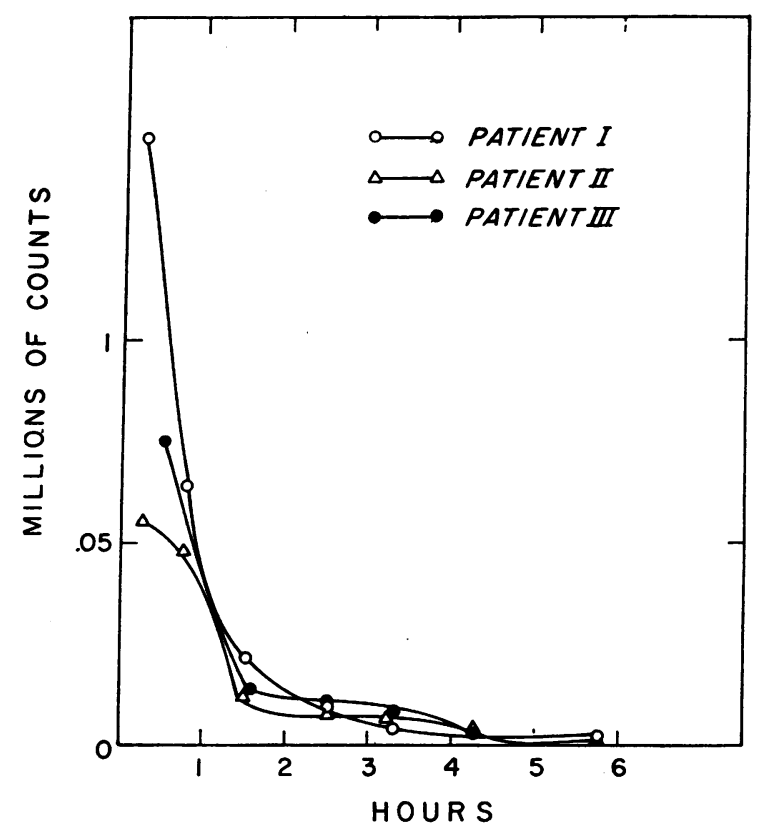

Fig. 5. Total COUNTS EXCRETEd IN URINE PER 15MINUTE INTERVAL AFTER THE ADMINISTRATION OF ISOTOPICALLY-LABELED OROTIC ACID. Observed counts not corrected for the 48 per cent efficiency of the counting instrument.

rates for orotic acid. The recovered orotic acid from the urine in period U 1 (Table I) had a specific activity of $1,401,000 \mathrm{cpm}$ per $\mu$ mole, whereas the administered orotic acid had a specific activity of 1,962,000 cpm per $\mu$ mole. Approximately $1.3 \mu$ moles $(0.2 \mathrm{mg})$ of orotic acid was presumably produced endogenously and excreted during this 30 -minute period. This would cor-

TABLE I

Patient $I$, minimal orotic acid clearances

\begin{tabular}{lccc}
\hline & Time & Flow rate & KOH digests \\
\hline & min & ml/min & total cpm in urine \\
U 1 & 31 & 5.54 & $6,050,000$ \\
U 2 & 30 & 5.07 & $1,290,000$ \\
U 3 & 30 & 6.37 & 430,000 \\
U 4 & 60 & 4.00 & 333,000 \\
& & & cpm in whole plasma \\
P 1 & 10 & & 4,401 \\
P 2 & 20 & & 1,959 \\
P 3 & 45 & & 855 \\
P 4 & 75 & & 388 \\
P 5 & 120 & & 225 \\
& Corotic aoid & & Coreatinine \\
& ml/min & & ml $/$ in \\
U 1 & 63 & & 79.1 \\
U 2 & 50.3 & & 94.1 \\
U 3 & 38 & & 91.0 \\
\end{tabular}



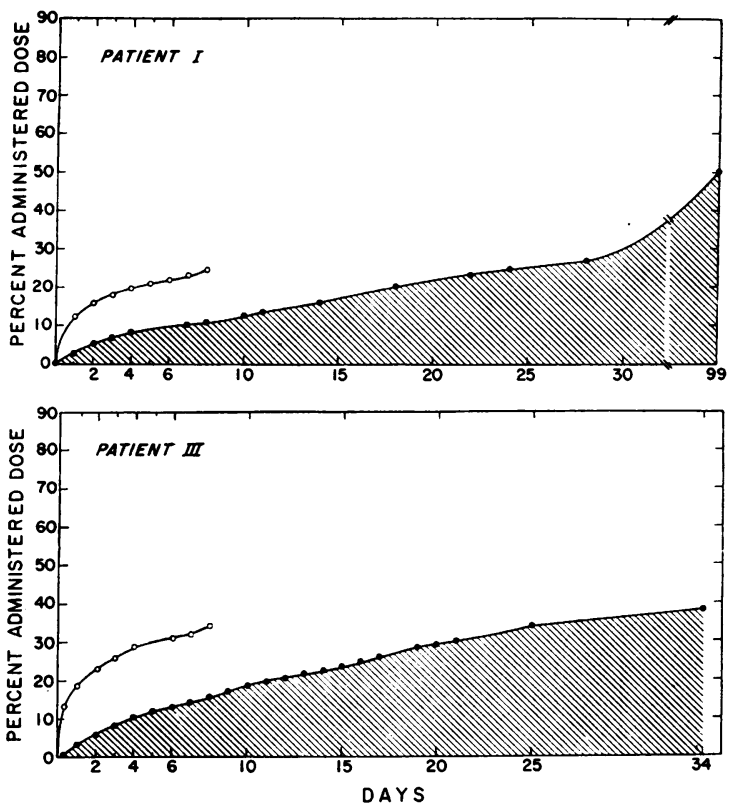

Fig. 6. RECOVERY OF ISOTOPE AFTER THE ADMINISTRATION OF ISOTOPICALLY-LABELED OROTIC ACID. Shaded area represents isotope recovered as respiratory $\mathrm{CO}_{2}$. Unshaded area between the two curves represents the additional radioactivity recovered in urine.

respond to the excretion of about $9.6 \mathrm{mg}$ of orotic acid per 24 hours in this patient on a purinefree diet.

Stool smears of the subjects were examined in a low-background counter, but there was never any detectable amount of radioactivity present. The sensitivity of the detection method was such that radioactivity would have been detected if over 1 per cent of the isotope had appeared in the feces in the first week.

The cumulative recovery of injected isotope in urine and expiratory $\mathrm{CO}_{2}$, presented for Patients I and III, is shown in Figure 6. With the previously mentioned extrapolations for urinary radioactivity, the recovery of isotope in these two subjects was approximately 80 and 65 per cent, respectively, by the end of the study.

An early peak ( 3 to 4.5 hours after the beginning of isotope injection) was seen in the specific activity curves of urinary uracil and respiratory $\mathrm{CO}_{2}$. This early peak was missing from the pseudouridine curve (Figures 1-4). Uracil specimens in the first few days had a specific activity several times higher than that of the corresponding pseudouridine specimens. Thereafter they de- clined more rapidly in specific activity, ultimately approaching that of pseudouridine. Because of the small amount of urinary uracil it was possible to obtain activities only for the first 1.5 to 2 weeks of the study, whereas pseudouridine could be followed for a considerably longer time. The specific activity curve for pseudouridine was also complex, particularly in Patient III, with more than one secondary rise.

\section{DISCUSSION}

Because of heretofore unknown radiation dosimetry, patients with neoplasia and limited life span were employed in this study. The excretion of pseudouridine at rates close to median normal values in this laboratory (4), as well as the clinically stable course of the patients, suggests that the data on these subjects are not grossly distorted as compared with data that might be obtained from a group of true normals.

When carboxyl-labeled orotic acid is given to animals or humans, most of the expired radioactivity appears as respiratory $\mathrm{CO}_{2}$ within a very few hours (11). The rapid disappearance of radioactivity from urinary orotic acid is consistent with this observation and suggests that intravenous administration of ring-labeled orotic acid provides something approaching "pulse-labeling" of newly formed pyrimidines in the body. The rate of excretion of isotope is quite consistent with reported experiments with ring-labeled orotic acid in the rat (12).

An approximate correction for nonutilized (spilled) radioactivity may be made by subtracting the number of counts found in the first three urine specimens (Figure 5) from the total counts injected.

The data obtained in these studies may be used to derive estimates of the rate of pyrimidine biosynthesis via orotic acid provided certain assumptions are made. Thus pyrimidine production rate may be calculated as: pseudouridine production rate $\times$ (total isotope utilized)/(total isotope incorporated into pseudouridine), if there does not exist a single population of cells that simultaneously and significantly violates the postulates A and $B$ which follow:

A. The ratio of pseudouridine production to total pyrimidine production is the same in each cell population as that for the whole body. 
B. The ratio of $\mathrm{C}^{14}$-orotic acid to total orotic acid utilized by these cells is the same as that for the entire body.

This calculation is further based on the assumption that orotic acid is not degraded, save by prior conversion to pyrimidine nucleotides, and is utilized intracellularly in the same manner as is endogenously produced orotic acid. The values calculated by this method for Patients I, II, and III are (all expressed as grams of pseudouridine per day) : I) $52 \mathrm{mg}$ pseudouridine per day $\times 31$ $=1.61$; II) $41 \mathrm{mg}$ per day $\times 30=1.23$; III) 50 $\mathrm{mg}$ per day $\times 23=1.15$. Pseudouridine excretion is considered to be equal to pseudouridine production (5). Total isotope incorporated into pseudouridine is estimated by linear extrapolation of the latter portion of the pseudouridine specific activity curves. Exponential extrapolation led to estimates of total pyrimidine production within 10 per cent of those obtained by linear extrapolation. These values are upper estimates that may be magnified by a slower decline of the final portion of the pseudouridine curve, or by pseudouridine production by intestinal bacteria and residual pseudouridine in the purine-free diet, or by both. The values are somewhat larger than are estimates of total purine production based on uric acid excretion (13), but are within the same order of magnitude. The estimated daily amounts of pyrimidine synthesis are substantially less than observed orotic acid excretion rates in patients treated with azauridine (7) or in a subject with orotic-aciduria (14).

Acid-soluble pyrimidine derivatives of tissues. after the administration to animals of isotopicallylabeled orotic acid, reach high levels of specific activity earlier than ribonucleic acid (12). The virtual absence of acid-soluble pseudouridine derivatives in the liver is quite probably related to the absence of the early peak in the pseudouridine specific activity curve. Presumably, the rate of incorporation of acid-soluble pseudouridine derivatives into ribonucleic acid is greater than the rate of dephosphorylation of these compounds in those cells actively engaged in pseudouridine synthesis.

The appearance of substantial amounts of radioactivity in urinary pseudouridine at approximately 12 hours suggests that there is a pseudouridine- bearing RNA which has a relatively rapid turnover rate. This might have some bearing on "shunt" mechanisms of uric acid synthesis (15), in that early overincorporation of isotopic precursors into uric acid could possibly occur through such a rapidly metabolized RNA as well as by the "shunting" of free nucleotides into uric acid.

The subsequent over-all rate of decline of urinary pseudouridine radioactivity represents an upper limit for the turnover of pseudouridinecontaining ribonucleic acids. It is unknown to what extent RNA turnover is an explosive process involving total breakdown of an individual RNA molecule to its constituent mononucleotide derivatives prior to their reutilization. If indeed this does occur, it is not known whether the fragments released are $5^{\prime}$ nucleotides, $2^{\prime}-3^{\prime}$ nucleotides, or other unidentified materials. In view of recent knowledge of RNA synthesis and ribonucleotide interconversions, $2^{\prime}-3^{\prime}$ nucleotides probably must be dephosphorylated before reincorporation into RNA (16). Under these circumstances, if cell penetration or intracellular channeling is not a limiting factor in pseudouridine utilization, the data previously obtained with labeled pseudouridine suggest that little re-utilization of pseudouridine would occur (5), and the decline of this curve would then be a true reflection of the "turnover" of this RNA.

The results in certain studies, particularly in Patient III, suggest that the declining portion of the pseudouridine curve is a composite consisting of several secondary rises in specific activity. In a general fashion, the specific activity curves for urinary uracil and respiratory $\mathrm{CO}_{2}$ tend to parallel each other, particularly in the early phase of the study.

The higher specific activity and more rapid decline in specific activity of uracil during the first five days of the study remain to be accounted for. Possible explanations include 1) the presence of a very rapidly renewing but pseudouridine-poor RNA, 2) relatively slowly renewing acid-soluble nucleotides, 3) a preferential utilization of exogenous orotic acid by cells not making pseudouridine-rich RNA, and 4) the possibility that the initial "spike" in the specific activity curve is due to direct decarboxylation of orotic acid or shunting of exogenous orotic acid-derived nucleotides 
separately from endogenously-made pyrimidine nucleotides, so that the second peak represents the true turnover of "acid-soluble" components. No evidence exists for any direct decarboxylation of orotic acid, and several negative experiments have been performed $(1,17,18)$. Nevertheless, further search for shunt mechanisms of orotic acid degradation is necessary before this last possibility can be firmly excluded.

\section{SUM M ARY}

Orotic acid labeled in ring positions with carbon ${ }^{14}$ was administered to four patients. Urinary radioactivity, respiratory carbon dioxide radioactivity, and urinary pseudouridine and uracil specific activities were measured. These data were considered in relation to renewal rates of ribonucleic acid pyrimidines. Estimates of the de novo rate of pyrimidine biosynthesis were derived for three patients.

\section{REFERENCES}

1. Reichard, P. Enzymic synthesis of pyrimidines. Advanc. Enzymol. 1959, 21, 263.

2. Adler, M., and Gutman, A. B. Uridine isomer (5ribosyluracil) in human urine. Science 1959, 130, 862.

3. Adams, W. S., Davis, F., and Nakatani, M. Purine and pyrimidine excretion in normal and leukemic subjects. Amer. J. Med. 1960, 28, 726.

4. Eisen, A. Z., Weissman, S. M., and Karon, M. Pseudouridine metabolism. I. Isolation and dietary effects. J. Lab. clin. Med. 1962, 59, 620.

5. Weissman, S. M., Eisen, A. Z., and Karon, M. Pseudouridine metabolism. III. Studies with isotopically labeled pseudouridine. In preparation.
6. Davidson, J. D., and Feigelson, P. Practical aspects of internal-sample liquid-scintillation counting. Int. J. appl. Radioisotopes 1957, $2,1$.

7. Fallon, H. J., Frei, E., III, Block, J., and Seegmiller, J. E. The uricosuria and orotic aciduria induced by 6-azauridine. J. clin. Invest. 1961, 40, 1906.

8. Gjone, E., Vance, H. G., and Turner, D. A. Direct liquid scintillation counting of plasma and tissues. Int. J. appl. Radiat. 1960, 8, 95.

9. Nathan, D. G., Davidson, J. D., Waggoner, J. G., and Berlin, N. I. The counting of barium carbonate in a liquid scintillation spectrometer. J. Lab. clin. Med. 1958, 52, 915.

10. Cohn, W. E. Pseudouridine, a carbon-carbon linked ribonucleoside in ribonucleic acids: Isolation, structure, and chemical characteristics. J. biol. Chem. 1960, 235, 1488.

11. Rabkin, M., Frederick, E., Lotz, M., and Smith, L. H., Jr. Pyrimidine biosynthesis in man: Suppression in vivo by anti-neoplastic agents (abstract). J. clin. Invest. 1961, 40, 1073.

12. Hurlbert, R. B., and Potter, V. R. A survey of the metabolism of orotic acid in the rat. J. biol. Chem. 1952, 195, 257.

13. Seegmiller, J. E., Grayzel, A. I., Laster, L., and Liddle, L. Uric acid production in gout. J. clin. Invest. 1961, 40, 1304.

14. Huguley, C. M., Jr., Bain, J. A., Rivers, S. L., and Scoggins, R. B. Refractory megaloblastic anemia associated with excretion of orotic acid. Blood 1959, 14, 615.

15. Wyngaarden, J. B., Seegmiller, J. E., Laster, L., and Blair, A. E. Utilization of hypoxanthine, adenine and 4-amino-5-imidazolecarboxamide for uric acid synthesis in man. Metabolism 1959, 8, 455.

16. Abrams, R. Nucleic acid metabolism and biosynthesis. Ann. Rev. Biochem. 1961, 30, 165.

17. Pasternak, C. A., and Handschumacher, R. E. The biochemical activity of 6-azauridine: Interference with pyrimidine metabolism in transplantable mouse tumors. J. biol. Chem. 1959, 234, 2992.

18. Weissman, S. M. Unpublished data. 